\title{
Mechanisms and mediators of lung injury after acute kidney injury
}

\section{Sarah Faubel and Charles L. Edelstein}

Abstract | Acute kidney injury (AKI) is a common complication in hospitalized patients, associated with $>50 \%$ mortality in those in intensive care who require renal replacement therapy. Data suggest that $\mathrm{AKI}$ is a systemic disease that adversely affects the immune system and organ function, and in this way contributes to the high mortality observed in affected patients. Data from patients and animal models indicate that $\mathrm{AKI}$ adversely affects the lungs. Respiratory complications are common in patients with $\mathrm{AKI}$ and include pulmonary oedema, respiratory failure requiring mechanical ventilation, prolonged duration of mechanical ventilation, and prolonged weaning from mechanical ventilation. The development of respiratory failure in patients with AKI greatly increases the risk of death. Data from animal models support the notion that cardiogenic pulmonary oedema (from volume overload) and non-cardiogenic pulmonary oedema (from endothelial injury due to inflammation and apoptosis) can occur in AKI. In this Review we discuss the clinical, epidemiologic, and animal data that provide insights into the mechanisms by which AKI can lead to lung injury and respiratory complications. Elucidation of the mechanisms of lung injury and respiratory complications after AKI is essential to develop effective therapies and reduce the high mortality associated with $\mathrm{AKI}$ and respiratory failure.

Acute kidney injury (AKI) occurs in $20 \%$ of hospitalized patients ${ }^{1}$ and $30-50 \%$ of admissions to the intensive care unit $(\mathrm{ICU})^{2}$. AKI is the most common in-patient consult to nephrologists, and is associated with marked morbidity and mortality ${ }^{3}$. The in-hospital mortality of patients with severe AKI requiring dialysis is $33 \%$ overall ${ }^{4}$ and $>50 \%$ in patients in the $\mathrm{ICU}^{5,6}$. Furthermore, patients who develop AKI remain in hospital longer and are twice as likely to be discharged to short-term or long-term care facilities than those who do not develop $\mathrm{AKI}^{7,8}$. The development of acute respiratory failure in patients with $\mathrm{AKI}$ increases the likelihood of discharge to an extended care facility ${ }^{8}$.

AKI has not always been considered to elicit adverse sequelae and was originally thought of as an acceptable consequence of hospital procedures ${ }^{9}$. Moreover, many clinicians assumed that patients die with AKI, rather than from AKI. A study published in 1996 challenged this complacency by showing that patients who developed AKI after intravenous administration of contrast agent had a 5.5-fold increased risk of in-hospital mortality compared to patients who did not develop $\mathrm{AKI}^{9}$. The patients with AKI also developed numerous non-renal complications, such as sepsis and respiratory failure, which accounted for this increased mortality risk. Following this publication, numerous well designed clinical studies demonstrated that AKI is independently associated with increased mortality ${ }^{9-15}$, even if the severity of AKI is considered to be relatively mild ${ }^{9,10,12}$. Consequently, clinicians and researchers now generally accept that patients can die from AKI and its deleterious effects ${ }^{16}$.

In this Review, we consider the complications and consequences of AKI that lead to increased mortality as either 'traditional' or 'non-traditional' (BOX 1). Traditional complications of AKI include fluid overload, bleeding, pericarditis, altered mental status, and electrolyte abnormalities, such as hyperkalaemia, acidosis, hyperphosphataemia, and hypocalcaemia ${ }^{17-22}$. These abnormalities have been recognized as complications of AKI for $>50$ years $^{23-26}$ and are the typical complications of AKI listed in textbooks $^{18,21}$, clinical practice guidelines ${ }^{19,22}$, and review articles ${ }^{20}$. Although fluid overload is associated with increased mortality in patients with $\mathrm{AKI}^{27-29}$, the very high mortality rate associated with AKI cannot solely be due to these traditional factors, particularly as these abnormalities can typically be corrected with dialysis and good supportive care. Since the 1950s (the early era of dialysis), nephrologists have observed that despite control of uraemic symptoms and normalization of electrolytes, patients with AKI may still die owing to the development of other complications, such as sepsis ${ }^{22,23}$. This unfortunate and frustrating observation continues today. Data from patients and animal models of AKI suggest that a 


\section{Key points}

- Respiratory complications are common after acute kidney injury (AKI) and are associated with increased mortality

- Cardiogenic (volume overload) and non-cardiogenic (lung injury with inflammation) pulmonary oedema can occur after AKI

- Animal data indicate that lung inflammation and lung endothelial apoptosis mediate non-cardiogenic pulmonary oedema after AKI

- Human and animal data suggest that IL-6, IL-8, TNF, NFkB, TNFR1 and caspase-3-mediated apoptosis, HMG-B1, and T cells mediate lung injury after AKI

- The high mortality associated with AKI might be due to both traditional (for example, electrolyte abnormalities) and non-traditional (for example, lung injury) complications of AKI much broader range of non-traditional complications likely account for the high mortality. In patients with AKI, these complications include sepsis ${ }^{9,30}$, heart failure $^{31}$, respiratory complications ${ }^{14,32}$, and immunoparalysis ${ }^{33}$ (BOX 1). Animal studies indicate that AKI causes lung ${ }^{34,35}$, $\operatorname{cardiac}^{36}$, liver ${ }^{37,38}$, brain ${ }^{39}$, and intestinal injury ${ }^{40}$. Unlike traditional complications, the non-traditional complications of AKI will probably require therapies beyond dialysis to correct them. Thus, data now support the notion that AKI should be considered a systemic disease that can have widespread deleterious effects on distant organs and immune function ${ }^{41-44}$.

Respiratory complications are a particularly important consideration in patients with AKI, and are the focus of this Review. Here, we discuss the clinical, epidemiologic, and pathologic mechanisms that underlie the respiratory complications associated with AKI. Although

\section{Box 1 | Traditional and non-traditional complications of AKI}

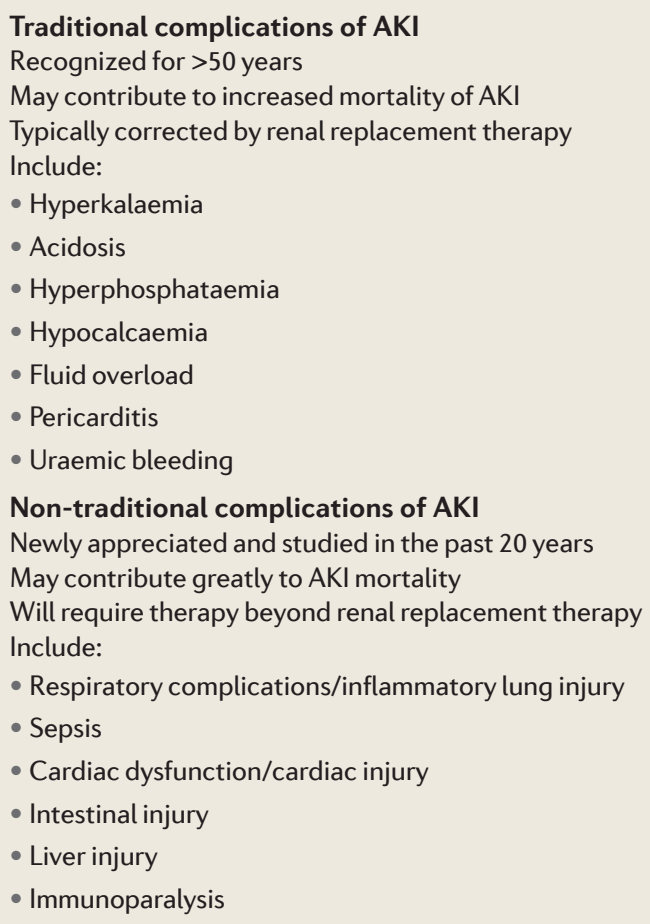

Abbreviation: AKI, acute kidney injury. our focus is the mechanisms by which AKI affects the lung, the association between kidney injury and lung injury is likely bidirectional, and primary injury to the lung might also cause injury to the kidney. For example, animal models have shown that AKI can cause acute lung injury $(\mathrm{ALI})^{45}$, and conversely, that ALI (particularly involving mechanical ventilation) can cause $\mathrm{AKI}^{46}$. Establishing such causality from clinical studies is challenging because the time of onset of AKI is often not known, and AKI is typically diagnosed (and studied) late in its course. As will be discussed, mediators of lung injury in AKI can increase in concentration in the serum before an increase in serum creatinine level is detected ${ }^{47}$. Establishing a time course of organ injury in patients is, therefore, challenging, rendering animal models particularly useful. An additional consideration when establishing the timing and cause of organ injury is that numerous conditions - particularly sepsis can lead to multiple organ failure that is caused by the underlying illness, and the role of individual organ failures on another organ can be difficult to determine. For example, the systematic examination of the onset of AKI and ALI in an animal model of sepsis indicated that AKI and ALI occurred simultaneously and were established $4 \mathrm{~h}$ after the induction of sepsis ${ }^{48}$.

\section{Respiratory complications in AKI}

Patients with AKI are twice as likely to develop respiratory failure requiring mechanical ventilation than are patients without $\mathrm{AKI}^{14,32}$, and the need for mechanical ventilation is even higher for patients with severe AKI that requires renal replacement therapy $(\mathrm{RRT})^{14}$. AKI with respiratory failure that requires mechanical ventilation is a considerable concern, as the requirement for mechanical ventilation is a consistent, independent predictor of mortality in patients with AKI ${ }^{14,32,49-56}$. One study found that the rate of death for patients with AKI requiring mechanical ventilation compared with that of patients not requiring mechanical ventilation was $81 \%$ and $29 \%$, respectively ${ }^{49}$. Furthermore, the occurrence of respiratory failure in patients with AKI portended the worst prognosis of all associated organ failures, with an odds ratio for mortality from AKI-associated respiratory failure of 10.3 versus 1.7 for AKI-associated non-respiratory organ failure $^{49}$. Using data from 1,122 patients with AKI requiring RRT, researchers from the large Veterans Affairs/NIH Acute Renal Failure Trial Network (ATN) developed a mortality prediction model. Mechanical ventilation and hypoxia without mechanical ventilation were the two factors of 21 variables with the highest points assigned in the mortality prediction model (where a higher score was associated with a higher overall risk of mortality $)^{57}$. Specifically, patients who were not on mechanical ventilation but required fractional inspired oxygen $\left(\mathrm{FiO}_{2}\right) \geq 60 \%$ were assigned 14 points and patients on mechanical ventilation with an $\mathrm{FiO}_{2}$ of $\geq 60 \%$ were assigned 12 points; all other factors that contributed to the mortality score carried a weight of $\leq 5$ points. In the parsimonious mortality prediction score derived from the ATN trial data, the inclusion of just four clinical factors were sufficient to predict mortality. These factors were requirement for 


\section{Box 2 Respiratory complications of AKI}

- Pulmonary oedema

Cardiogenic (hydrostatic, from fluid overload and/or cardiac dysfunction)

Non-cardiogenic (endothelial injury from inflammation; endothelial cell death from apoptosis)

- Impaired lung fluid clearance (decreased lung epithelial sodium channel and lung aquaporins)

- Respiratory failure requiring mechanical ventilation Prolonged duration of mechanical ventilation Prolonged weaning from mechanical ventilation

Abbreviation: AKI, acute kidney injury.

mechanical ventilation, increasing age, increasing levels of bilirubin, and lower mean arterial pressure ${ }^{58}$. Thus, respiratory complications are a key marker of mortality risk in patients with AKI, suggesting a unique association between AKI and respiratory complications. This paradigm is especially true as the overall mortality of ALI is $<20 \%{ }^{59}$, whereas the overall mortality of AKI requiring RRT in the ATN trial was $>60 \%$. Clinical data also suggest that AKI might have an effect on the recovery of lung function as patients with AKI require mechanical ventilation for a longer period of time and wean from mechanical ventilation over a longer period of time than comparably ill patients without $\mathrm{AKI}^{60}$.

\section{Cardiogenic pulmonary oedema in AKI}

Respiratory complications in AKI are predominantly caused by the development of pulmonary oedema (BOX 2), which can be cardiogenic or non-cardiogenic (FIG. 1). Fluid overload that results in hydrostatic (cardiogenic) pulmonary oedema has long been recognized as a cause of respiratory failure after $\mathrm{AKI}^{24,61-63}$. In paediatric patients at high risk of AKI, achievement of a net even fluid balance with peritoneal dialysis at the time of cardiac surgery resulted in $\sim 25 \%$ fewer hours of mechanical ventilation ${ }^{60}$. In general, fluid overload is associated with higher mortality in patients with $\mathrm{AKI}^{28,64}$, with one study finding the adjusted odds ratio for mortality in patients with AKI and fluid overload at the time of initiating RRT to be 2.07 (95\% CI 1.27-3.37) compared to patients with AKI without fluid overload $^{65}$. Cardiogenic pulmonary oedema can occur in patients with normal or impaired cardiac function, and is characterized by an increase in jugular and central venous pressure, and bilateral pulmonary infiltrates that can be detected by chest radiography (FIG. 1). Increased hydrostatic pressure results in the extravasation of fluid from the capillaries into the interstitial lung space, which is usually treated successfully with diuresis in patients with adequate kidney function, or ultrafiltration.

\section{Non-cardiogenic pulmonary oedema in AKI}

Non-cardiogenic pulmonary oedema occurs when the lung endothelium is injured, allowing the direct passage of fluid out of the capillaries into the interstitium. In the presence of epithelial and endothelial injury, fluid can fill the alveolar space and cause alveolar pulmonary oedema. The oedema fluid is generally proteinaceous and can be identified by routine pink eosin histologic staining. Measures of heart function, central venous pressure, and pulmonary capillary wedge pressure are normal ${ }^{66}$. As with cardiogenic pulmonary oedema, bilateral infiltrates can be detected by chest radiography; therefore, this method cannot be used to reliably distinguish between cardiogenic pulmonary oedema and non-cardiogenic pulmonary oedema (FIG. 1).

Pulmonary oedema in the presence of normal or low pulmonary capillary wedge pressure has been demonstrated in small studies of patients with $\mathrm{AKI}^{67,68}$, supporting the proposal that non-cardiogenic pulmonary oedema can occur in AKI. The role of proinflammatory mediators in the accumulation of neutrophils in the lung is central to the pathogenesis of endothelial and epithelial injury that leads to non-cardiogenic pulmonary oedema in ALI. Non-cardiogenic oedema is, therefore, typically caused and characterized by increased markers of inflammation and neutrophil accumulation, which will be discussed in more detail below ${ }^{69}$. Autopsy examination of patients with AKI has clearly demonstrated the occurrence of lung neutrophil infiltration associated with pulmonary oedema ${ }^{70,71}$. Proteinaceous oedema has been identified as a feature of lung oedema in patients with $\mathrm{AKI}^{70,72,73}$, including in the largest case series to date that compared the autopsy findings of 107 patients with AKI to those of 429 patients without $\mathrm{AKI}^{71}$. Notably, other features of ALI were also found in these autopsy series, including hyaline membranes, leading to the conclusion by some investigators that AKI might be considered a specific cause of $\mathrm{ALI}^{74}$. Taken together, these clinical data support the notion that AKI causes inflammation, endothelial damage, non-cardiogenic pulmonary oedema, and features of ALI (FIG. 2).

Recruitment of neutrophils to the lung, leading to non-cardiogenic pulmonary oedema in ALI is generally considered a consequence of the elaboration of proinflammatory mediators that cause systemic inflammatory response syndrome (SIRS). SIRS is characterized by the early production and release of proinflammatory cytokines in response to sepsis, pancreatitis, and other systemic (indirect) causes of ALI. Data from patients with AKI indicate that AKI is also associated with a SIRS response and that serum levels of the proinflammatory cytokines IL- $6^{47,75-78}$, IL- $8^{47,58,75,78,79}$, and TNF $^{75}$ are increased. Studies suggest that proinflammatory cytokines can induce lung injury by directly injuring the lung endothelium and causing non-cardiogenic pulmonary oedema. This process is achieved via the upregulation of pulmonary adhesion molecules and chemokines, which can promote neutrophil trapping in the endothelium, infiltration, and subsequent tissue damage (FIG. 3) $)^{80-85}$.

\section{Spectrum of pulmonary oedema in AKI}

Vigorous debate has been ongoing since the 1930s (when the radiographic term "uraemic oedema" was first coined $^{72}$ ) as to whether pulmonary oedema as a result of 

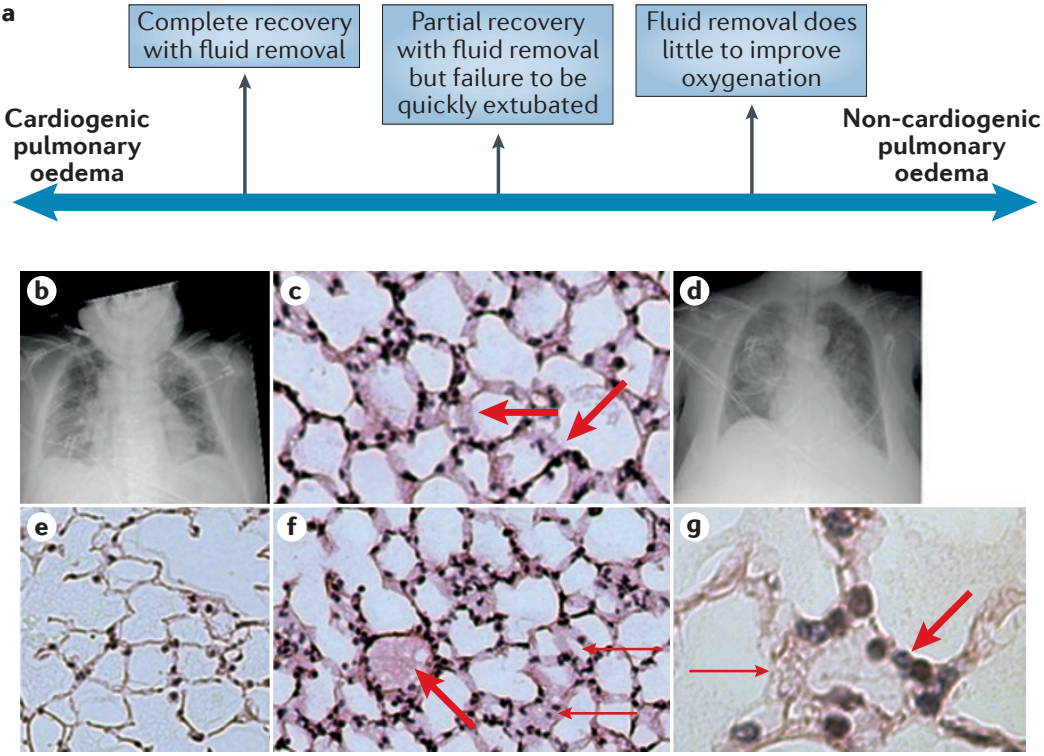

Figure 1 | Pulmonary oedema in AKI. a | Spectrum of pulmonary oedema in AKI. Cardiogenic pulmonary oedema is caused by fluid overload and/or cardiac dysfunction, leading to increased capillary hydrostatic pulmonary pressure and transudative pulmonary oedema. Patients can improve quickly with fluid removal via ultrafiltration or diuresis. Non-cardiogenic pulmonary oedema is caused by injury to the capillary membrane, leading to increased leak and proteinacious pulmonary oedema. Patients improve little or not at all with fluid removal via ultrafiltration or diuresis. Many patients likely fall in the spectrum between cardiogenic and non-cardiogenic pulmonary oedema. b Cardiogenic pulmonary oedema is characterized by bilateral infiltrates visible after chest radiography, and is generally without histologic or biochemical evidence of inflammation. Accurate measurements of central venous pressure or pulmonary artery occlusion pressure (PAOP; previously known as pulmonary capillary wedge pressure) are increased. c| Histologic image showing interstitial oedema (arrows). $\mathbf{d}$ | Non-cardiogenic pulmonary oedema is characterized by bilateral infiltrates visible after chest radiography, as well as histologic or biochemical evidence of inflammation. Accurate measurements of central venous pressure of PAOP are usually normal or low. e | Normal lung histology. $\mathbf{f} \mid$ The histologic image shows proteineaceous oedema (thick arrow) and interstitial oedema (thin arrows). g| The magnified histologic image shows neutrophils in interstitial space (thick arrow) and interstitial oedema (thin arrow) typical of non-cardiogenic pulmonary oedema.

AKI was caused by fluid retention or capillary injury as a unique consequence of renal failure and an accumulation of injurious uraemic toxins. As an example of this spirited debate, a report published in 1956 in the Journal of the American Medical Association opened with the statement, "The purpose of this paper is to deny the existence of the roentgenographic entity known as 'pulmonary azotemia', 'uraemic lung', or 'uraemic pneumonia"'s6. The authors of this paper seemed to consider that fluid overload alone was responsible for pulmonary oedema in AKI. Today, the accumulated evidence clearly suggests that either cardiogenic or non-cardiogenic pulmonary oedema can occur in AKI. Rather than attempting to define patients as having one form of oedema or another, we suggest it might be more useful to view pulmonary oedema in patients with $\mathrm{AKI}$ as existing on a spectrum between cardiogenic and non-cardiogenic, such that some patients might have a pure form of one or the other, but that many (if not most) patients with AKI and respiratory failure will have a combination of both forms of oedema (FIG. 1). Clinical experience and evidence ${ }^{62,86}$ supports the notion that a subset of patients with hypoxaemia or respiratory failure that requires mechanical ventilation seem to recover completely with fluid removal, suggesting that fluid overload leading to cardiogenic oedema was the primary cause of the respiratory failure in these patients. Other patients might recover somewhat with fluid removal but fail to be quickly extubated, and likely exhibit both cardiogenic and non-cardiogenic pulmonary oedema. Finally, patients in whom fluid removal does little to improve oxygenation might be considered to have predominantly non-cardiogenic pulmonary oedema. A bidirectional association between cardiogenic pulmonary oedema and non-cardiogenic pulmonary oedema probably exists, with one form able to cause or exacerbate the other. For example, hydrostatic pulmonary oedema is known to cause inflammation ${ }^{87}$ and likely exacerbates established ALI. Clinical trials have supported this notion, and have demonstrated that avoidance of fluid overload in ALI leads to better pulmonary outcomes than outcomes associated with fluid overload ${ }^{88}$.

\section{Kidney injury versus dysfunction}

The current approach to understanding the deleterious systemic effects of AKI has been to identify injurious circulating factors that accumulate with impaired kidney function and those that accumulate with kidney injury (FIG. 2). Loss of kidney function can cause an acute accumulation of substances that are normally cleared by the kidney, which might subsequently mediate deleterious systemic effects ${ }^{89}$. In contrast, kidney injury might lead to the release of numerous factors associated with cell injury and death that include, but are not limited to, cytokines, necrotic debris, microparticles, DNA, RNA, and microRNA. This effect is especially true in cases of renal ischaemia-reperfusion injury. Distinguishing between the deleterious systemic effects of loss of kidney function and kidney injury has been attempted experimentally by comparing the effects of bilateral nephrectomy (pure loss of kidney function) with ischaemic AKI (loss of kidney function with ischaemic kidney injury) (FIG. 2). Separating the systemic effects of loss of kidney function from kidney injury in clinical studies might be achieved by comparing the outcomes of similarly ill patients with either end-stage renal disease (ESRD) or AKI. The results of such studies provide interesting insights as to how the loss of kidney function (in both ESRD and AKI) versus kidney injury (AKI only) can affect outcomes in general, as well as respiratory complications specifically, as will be discussed below.

\section{Mortality in AKI and ESRD}

A study that compared in-hospital mortality among critically ill patients in the ICU reported death rates for patients with neither AKI nor ESRD, patients with ESRD, patients with $\mathrm{AKI}$, and patients with AKI requiring RRT of $5 \%, 11 \%, 23 \%$ and $58 \%$, respectively ${ }^{90}$. In this study, the severity of illness was judged using the APACHE III (Acute Physiology and Chronic Health Evaluation III) classification system ${ }^{91}$ and was deemed similar among all groups. Severity of illness, therefore, could not explain the marked increase in mortality in patients with AKI (with or without need for RRT) versus ESRD. The higher mortality 


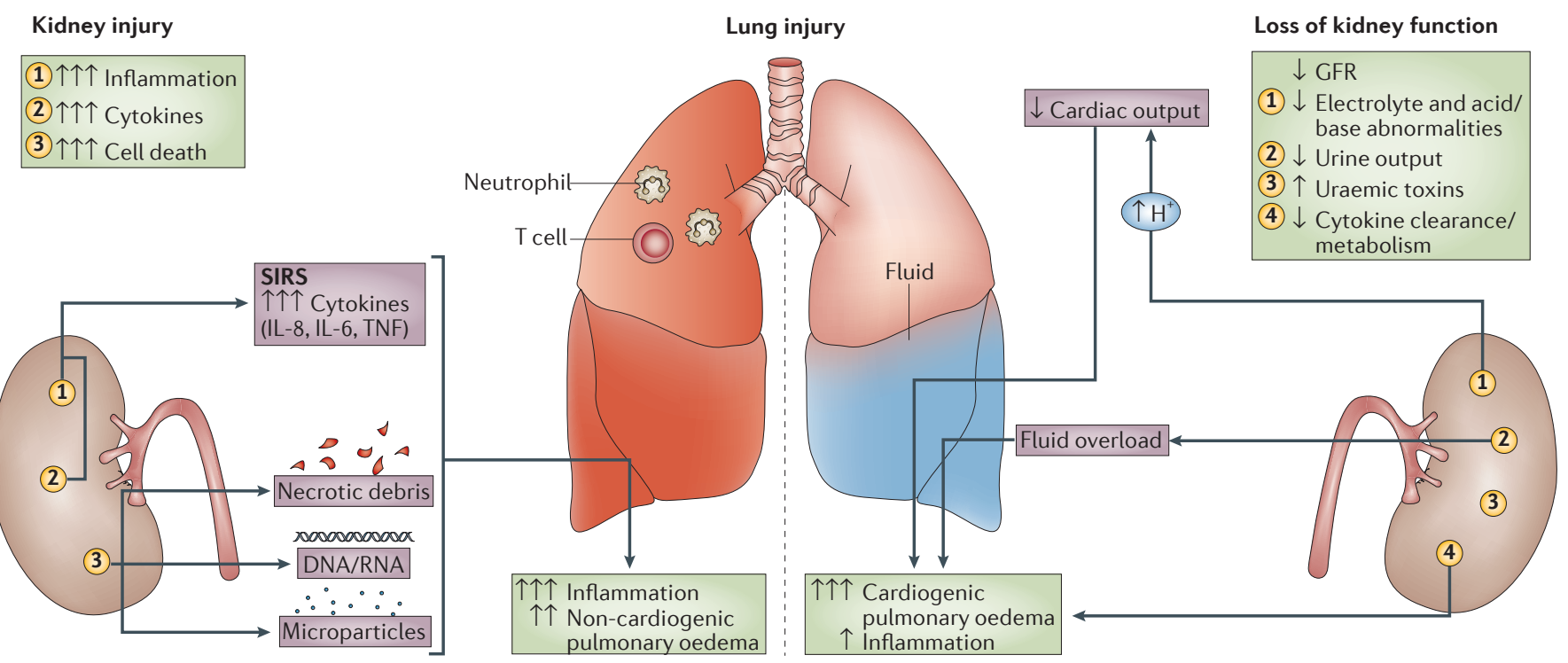

Figure 2 | The effects of kidney injury and kidney failure on the lung. The effects of acute kidney injury on the lung can be conceptually divided into factors that are associated with kidney injury and factors that are associated with loss of kidney function. Kidney injury leads to a robust inflammatory response, in part due to cell death, that is characterized by increased production and release of inflammatory mediators such as TNF, IL-6, and IL-8, which are known mediators of lung injury after AKI. Other possible factors that might be present in the circulation due to kidney injury and cell death include other proteins, DNA, RNA, and microparticles. Loss of kidney function (modelled by bilateral nephrectomy in animals) results in the accumulation of factors normally excreted and metabolized by the kidney that could affect lung function. Loss of kidney function can affect the lung if reduced fluid excretion occurs resulting in fluid retention, volume overload and hydrostatic (cardiogenic) pulmonary oedema. Certain electrolyte abnormalities can also contribute, including acidosis which might lead to cardiogenic pulmonary oedema by impairing cardiac output. The role of typical uraemic toxins - such as those that accumulate in end-stage renal disease — remains to be determined. Finally, since the kidney contributes to cytokine elimination, known inflammatory mediators (for example, IL-6) might accumulate to a greater extent in patients with kidney failure than in patients with normal kidney function. Abbreviations: GFR, glomerular filtration rate; SIRS, systemic inflammatory response syndrome.

of patients with AKI compared to patients with ESRD has been consistently observed ${ }^{90,92-95}$. Many studies, however, have included a heterogeneous population of patients with AKI that required any form of RRT (intermittent or continuous ${ }^{90,92,93}$ ) or no RRT at all ${ }^{90}$. We are aware of two studies that specifically examined mortality in critically ill patients with ERSD or AKI and who required the same modality of RRT, namely continuous RRT. In one of these studies the death rate was $63 \%$ for AKI and $46 \%$ for $\mathrm{ESRD}^{94}$ and in the other, the death rate was $61 \%$ for AKI and $36 \%$ for $\mathrm{ESRD}^{95}$. Interestingly, the lower mortality of patients with ESRD was evident despite the fact that these patients were older and had additional comorbidities, such as hypertension or diabetes mellitus ${ }^{95}$. These data support the hypothesis that distinct factors are associated with the acute loss of kidney function and kidney injury that contribute to mortality (FIG. 2).

\section{Mechanical ventilation in AKI and ESRD}

Five studies have specifically compared outcomes in AKI and ESRD ${ }^{90,92-94}$, three of which identified statistically meaningful factors that were associated with increased mortality in patients with $\mathrm{AKI}^{92,93,95}$. Notably, all three reports showed that the need for mechanical ventilation was higher in patients with AKI compared to those with ESRD, and that the need for mechanical ventilation was an independent risk factor for mortality ${ }^{92,93,95}$. In one study, the need for mechanical ventilation was the single driving factor associated with increased mortality, with $89 \%$ of patients with AKI requiring mechanical ventilation compared to $57 \%$ of patients with ESRD, conferring an odds ratio of 3.4 for increased mortality ${ }^{95}$.

The greater need for mechanical ventilation in patients with AKI compared to patients with ESRD with a similar severity of illness suggests that factors that are specifically associated with AKI predispose to respiratory failure. These studies reveal two potential factors by which AKI can predispose patients to respiratory failure - more aggressive fluid resuscitation in patients with $\mathrm{AKI}$ and a more severe systemic inflammatory response in AKI (FIG. 3) ${ }^{95}$. Although percentage fluid overload was not determined, patients with AKI had a significantly greater body weight at the initiation of continuous RRT compared to those with ESRD, which likely reflects fluid overload $^{95}$. As fluid management in patients with AKI is generally more liberal than in patients with ESRD, fluid overload leading to cardiogenic pulmonary oedema might occur more commonly in patients with AKI than in those with ESRD ${ }^{61}$. Notably, patients with ESRD are known to have a higher BMI and an increased likelihood of obesity than the general population ${ }^{96}$, suggesting that the marked difference in weight between those with AKI and ESRD at initiation of continuous RRT reflects a greater fluid overload status in patients with AKI. 


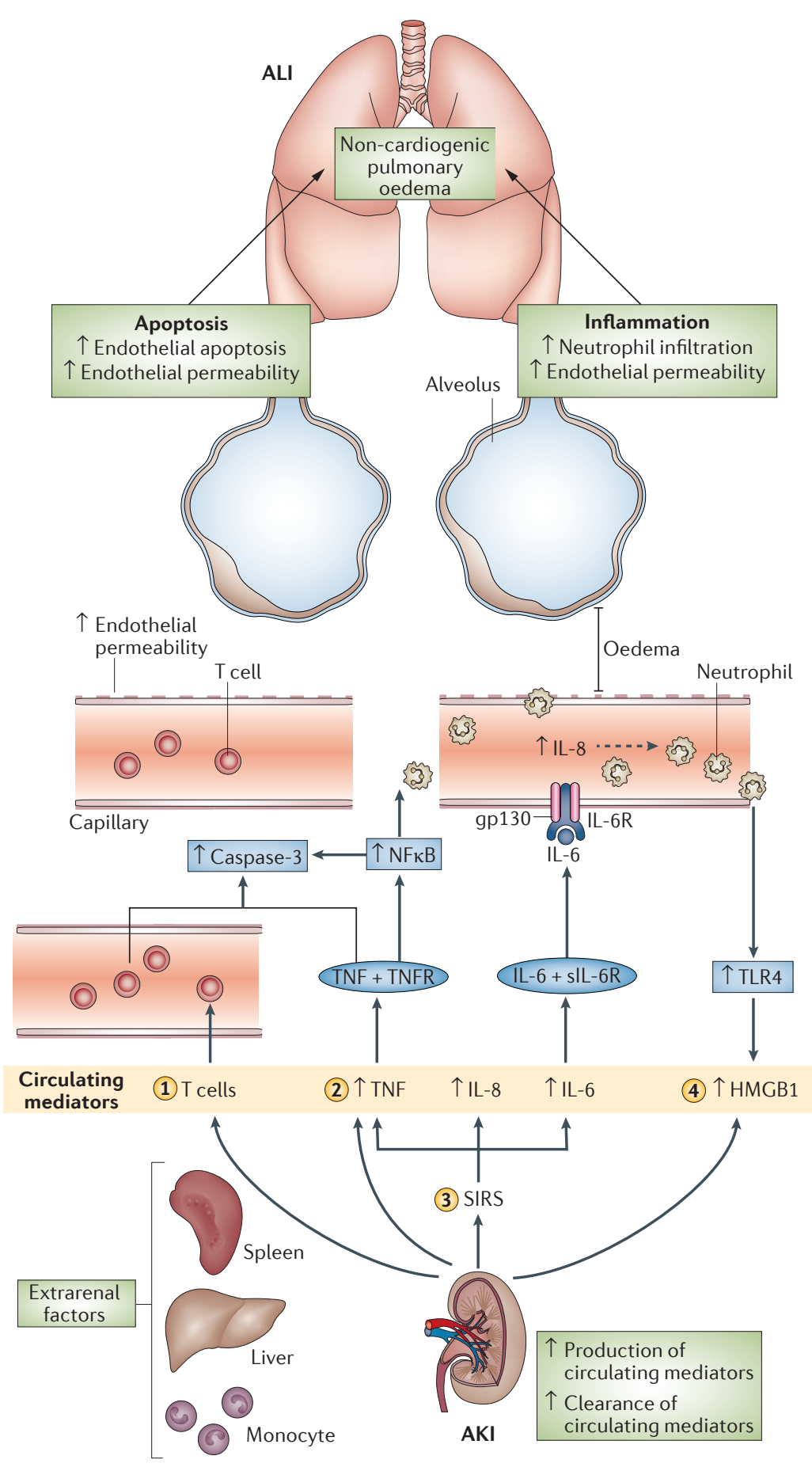

Patient data have demonstrated that AKI is associated with a proinflammatory phase and SIRS response as judged by increased serum IL- $6^{47,75-78}$, IL- $8^{47,58,75,78,79}$, and $\mathrm{TNF}^{75}$ (FIG. 3) ${ }^{97}$. Conversely, patients with ESRD are generally considered immunosuppressed ${ }^{98}$ and proinflammatory cytokine production might be dampened in these patients (FIG. 2). Consequently, patients with ESRD might exhibit relative protection from ALI and other complications associated with SIRS. Serum albumin, a negative acute phase reactant that is reduced as a consequence of SIRS, was shown in one study to
4 Figure 3 | Cascade of events leading from AKI to ALI. T cells are recruited to the lung and initiate caspase-3-mediated apoptosis of lung endothelial cells, which results in increased lung capillary permeability and non-cardiogenic pulmonary oedema (1). TNF acts via TNFR1 on lung endothelial cells resulting in endothelial cell apoptosis via caspase- 3 activation, which causes increased lung capillary permeability and non-cardiogenic pulmonary oedema (2). TNF also activates NFkB which then increases both inflammation and apoptosis leading to endothelial injury, increased lung capillary permeability, and non-cardiogenic pulmonary oedema. AKI is associated with an increase in serum levels of proinflammatory cytokines such as IL-6, IL-8, and TNF, which is consistent with a SIRS response (3). Proinflammatory cytokines are increased in AKI due to their increased renal production, increased extrarenal production (for example, in the monocytes, liver, and spleen), decreased renal clearance, and decreased renal metabolism. Circulating IL-6 binds to circulating soluble IL-6R to engage gp130 on endothelial cells and increase production of IL-8. IL-8 production causes lung injury by facilitating neutrophil accumulation which leads to endothelial injury, increased lung capillary permeability, and non-cardiogenic pulmonary oedema. HMGB1 leads to TLR4 activation (4). The net effect is inflammation, neutrophil accumulation, endothelial injury, increased lung capillary permeability, and non-cardiogenic oedema. Abbreviations: AKI, acute kidney injury; ALI, acute lung injury; SIRS, systemic inflammatory response syndrome.

be markedly lower in patients with AKI compared to patients with ESRD ${ }^{95}$. Another study examined the plasma metabolome (metabolites, plasma proteome, and whole blood transcriptome) of critically ill patients with SIRS and found marked differences between patients with AKI, ESRD, and normal renal function ${ }^{99}$. Mortality was lowest in patients with ESRD. Notably, the transcriptome analysis revealed that $98.5 \%$ of $\sim 2,000$ transcripts examined were decreased in patients with ESRD compared to levels in individuals with normal kidney function. These data further support the notion that reduced production of injurious mediators might occur in ESRD and provide relative protection from ALI and other deleterious effects of the SIRS response ${ }^{99}$.

\section{Ischaemic AKI and bilateral nephrectomy Lung injury}

Animal models of ischaemic AKI and bilateral nephrectomy have been invaluable in characterizing the lung injury that occurs after AKI. In order to understand the nature of lung injury that has been observed in animal models of AKI, we must first clarify the definition of ALI in animal models. Based on the official workshop and consensus report conducted and approved by the American Thoracic Society, ALI is considered to be present in small animals (such as mice and rats) if three of the four following features are present: histologic evidence of tissue injury; pulmonary oedema due to alteration of the alveolar capillary barrier (non-cardiogenic pulmonary oedema); inflammation; physiologic dysfunction ${ }^{100}$. Numerous assessments can be performed to document these features, and measurements have 
been categorized by the consensus group as either "very relevant" or "somewhat relevant". Examples of very relevant histologic injury include an increased number of neutrophils in the alveolar or interstitial space, or increased injury based on standardized histologic scoring. An increase in bronchoalveolar lavage (BAL) fluid protein, albumin, or IgM is considered a very relevant indicator of non-cardiogenic pulmonary oedema. Increased BAL fluid albumin, total protein, or IgM usually suggests that injury to the alveolar capillary barrier has occurred, thus enabling the entry of these high molecular weight compounds into the alveolar space ${ }^{100}$, although exceptions exist ${ }^{87,101,102}$.

Very relevant measures of lung inflammation include high levels of neutrophils in BAL fluid; high lung myeloperoxidase activity; and a high level of lung tissue or BAL fluid proinflammatory cytokines ${ }^{100}$. Finally, hypoxaemia is considered a very relevant measurement of physiologic dysfunction. In animal models of AKI, markers of histologic injury, capillary leak, and inflammation have been demonstrated, but no model has documented a change in oxygenation ${ }^{103}$. Three of the four criteria have, however, been met in models of AKI and satisfy the agreed criteria to represent ALI in model systems.

\section{Lung inflammation}

Numerous anti-inflammatory agents protect against lung injury after ischaemic AKI or bilateral nephrectomy, which supports the role of inflammatory mediators in lung injury. The list of protective anti-inflammatory agents includes CNI-1493, an inhibitor of the p38 MAP kinase pathway ${ }^{35}$; $\alpha-\mathrm{MSH}^{104}$; IL-6 inhibitors (anti-IL-6 antibodies or through genetic deficiency) ${ }^{105}$; IL-10 ${ }^{89}$; IL-8 inhibitors (via anti-IL-8 antibodies or through genetic deficiency of the IL- 8 receptor) ${ }^{106}$; dexmedetomidine (an anti-inflammatory anaesthetic agent ${ }^{107}$; anti-HMGB1 antibodies ${ }^{108}$; TLR4 inhibitors (via inhibitory mutations) ${ }^{108}$; NFkB inhibitors ${ }^{109}$; and entanercept (an inhibitor of TNF) ${ }^{109}$.

Lung inflammation is a consistent finding after ischaemic AKI, and is evaluated by increased neutrophil accumulation and protein and gene expression of proinflammatory mediators. Neutrophil accumulation in the lungs occurs within 1-2 h of onset of ischaemic $\mathrm{AKI}^{105}$, peaks at $4-8 \mathrm{~h}^{104,105,107}$, and although still elevated, neutrophil levels return to near baseline levels by $24-36 h^{105}$. One study examined the specific location of neutrophil accumulation in the lungs and found accumulation along the lung endothelium within $2 \mathrm{~h}$ of the onset of ischaemic AKI ${ }^{110}$. Margination and trapping of neutrophils in the endothelium occurs in animal models of ALI and is considered central to its pathogenesis in patients ${ }^{111}$. Inflammatory mediators that are increased in the lung after ischaemic AKI include TNF ${ }^{104,107}$, TNFR $1^{109}$, adhesion molecules

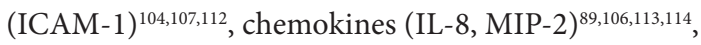
and $\mathrm{NF} \kappa \mathrm{B}^{104,109}$. Gene chip analyses of inflammatory pathways in the lungs have provided interesting results regarding the molecules that are modulated as a result of ischaemic AKI. A study of 109 inflammatory genes were examined in the lung at 6 and $36 \mathrm{~h}$ after ischaemic $\mathrm{AKI}^{115}$. Although numerous genes were upregulated, functional genomic analyses revealed that the IL- 6 and IL-10 signalling pathways were involved in the adverse effects of ischaemic AKI on the lung. IL-6 signalling is likely an injurious response and IL-10 signalling a protective response, as strategies to inhibit IL-6 protect against lung injury in $\mathrm{AKI}^{113}$ and lack of IL-10 exacerbates lung injury after ischaemic $\mathrm{AKI}^{114}$.

Many, but not all ${ }^{34,103}$, studies have found that bilateral nephrectomy is also associated with accumulation of lung neutrophils ${ }^{89,105,108,116}$, and an increase in proinflammatory mediators such as IL-8 ${ }^{105,108}$, CXCL2 ${ }^{116}$, $\mathrm{CINC} 2^{116}$, IL- $1 \beta^{108}$, and TNF ${ }^{108}$. In general, however, we consider it fair to state that lung inflammation after bilateral nephrectomy is less severe than that which occurs after ischaemic AKI on the basis of levels of inflammatory mediators and neutrophil markers. This notion suggests that kidney failure with kidney injury causes lung injury to a greater extent than does kidney failure alone.

\section{Pulmonary oedema}

As discussed, numerous studies have found that pulmonary oedema occurs after either ischaemic AKI or bilateral nephrectomy, as judged by an increased wet:dry lung weight ratio ${ }^{104,107,117}$, increased BAL fluid protein $^{34,89,117-119}$, and increased Evans blue dye accumulation in the lung ${ }^{34,35,108,113,116}$. Other studies, however, have failed to demonstrate this effect ${ }^{34,103,110,120}$. An important consideration with regard to the development of pulmonary oedema is the clearance mechanism of lung fluid that is dependent on lung sodium channels, Na-K ATPase, and aquaporins ${ }^{17,121}$. Decreased expression of the lung epithelial sodium channel ${ }^{117,121}$, lung $\mathrm{Na}-\mathrm{K}$ ATPase ${ }^{121}$, lung aquaporin-5 $5^{121}$, and lung aquaporin- $1^{117}$ has been shown in AKI, supporting the concept that impaired lung fluid clearance mechanisms could contribute to pulmonary oedema.

\section{Lung gene profiling}

Although lung neutrophil accumulation and pulmonary oedema occur after ischaemic AKI or bilateral nephrectomy, data suggest that the lung response to these two forms of AKI might be vastly different. In one study, $>22,000$ genes were examined in lung tissue $6 \mathrm{~h}$ or $36 \mathrm{~h}$ after either ischaemic AKI or bilateral nephrectomy ${ }^{34}$. At $6 \mathrm{~h}, 266$ genes were increased and 615 genes were decreased after ischaemic AKI. No genes were changed $6 \mathrm{~h}$ after bilateral nephrectomy, but hundreds of genes were affected $36 \mathrm{~h}$ after ischaemic AKI or bilateral nephrectomy. Notably, 600 genes were elevated following ischaemic AKI and 226 genes were elevated after bilateral nephrectomy at $36 \mathrm{~h}$, with 145 genes common to both. A total of 327 genes were decreased in expression after ischaemic AKI and 293 genes were decreased after bilateral nephrectomy, with 123 genes common to both. Further analyses of the affected gene pathways revealed marked activation of proinflammatory and pro-apoptotic processes, as will be discussed in more detail below. This study 
Table 1 | Mediators of lung injury after AKI

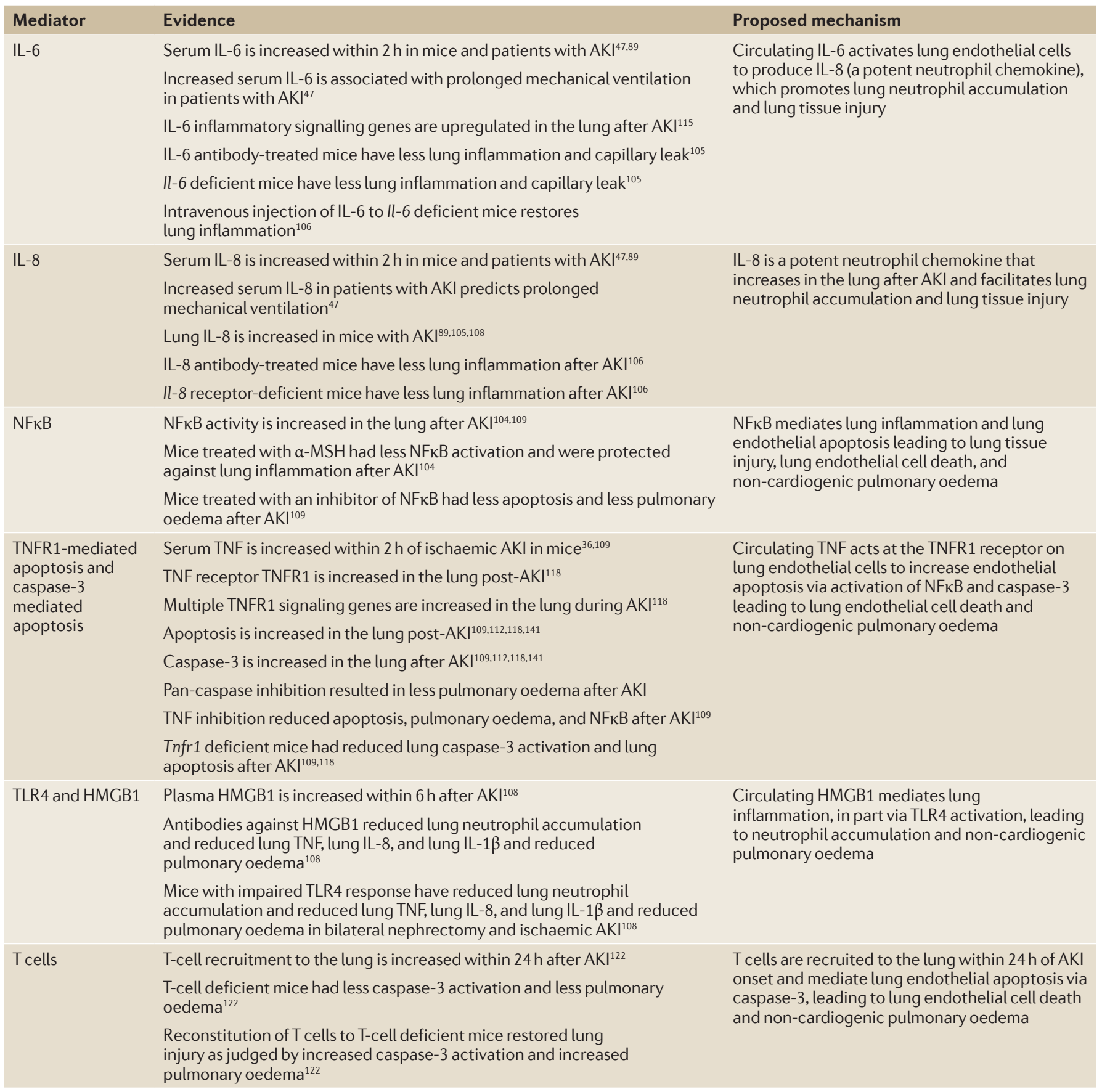

Abbreviation: AKI, acute kidney injury.

confirmed that transcriptional alterations that occur in the lung differ between ischaemic AKI and uraemia (bilateral nephrectomy) alone.

\section{Mediators of lung injury after AKI}

Accumulating evidence from animal models and patients with AKI suggests that IL-6, IL-8, TNF, NFkB, TNFR1 and caspase-3-mediated apoptosis, HMGB1, and $\mathrm{T}$ cells are mediators of lung injury after AKI (TABLE 1; BOX 3). The most well studied mediators of lung injury after AKI, namely IL-6, TNFR1 and caspase- 3 mediated apoptosis, and $\mathrm{T}$ cells $\mathrm{s}^{122,123}$ are discussed in detail below.

\section{IL-6 in AKI-mediated lung injury}

Elevated serum levels of IL- 6 can be used to predict the development of AKI under various conditions, including after cardiopulmonary bypass surgery ${ }^{47}$, in sepsis ${ }^{76}$, and in ALI $^{124}$. Serum IL-6 levels are increased $2 \mathrm{~h}$ after AKI caused by cardiopulmonary bypass surgery, and are associated with prolonged mechanical ventilation ${ }^{47}$. Furthermore, increased serum IL-6 levels are associated 


\section{Box 3 | Indicators of lung injury after acute kidney injury}

Very early (0-6h)

Increased serum proinflammatory cytokines (for example, IL-6, IL-8, TNF)

Increased serum mediators (for example, HMGB1)

Increased lung NFKB

Increased lung chemokines (for example, MIP-2 and IL-8)

Increased lung adhesion molecules (for example, ICAM-1)

Increased lung markers of inflammation (for example, IL-1 $\beta$ )

Pulmonary oedema

Lung neutrophil accumulation

Early (24h)

Lung T-cell accumulation

Increased lung endothelial apoptosis, caspase 3 activity, and lung TUNEL staining

Pulmonary oedema

Lung neutrophil accumulation

Late (7 days)

Increased lung IL-8

Lung neutrophil accumulation

Abbreviation: TUNEL, terminal deoxynucleotidyl transferase dUTP nick end labelling. with increased mortality in patients with established $\mathrm{AKI}^{75}$ and $\mathrm{ALI}^{125,126}$.

As discussed, inhibition of IL- 6 protects against AKI-mediated lung injury ${ }^{113}$. Specifically, IL-6 deficient mice and mice treated with IL-6 antibodies exhibit reduced lung inflammation, reduced capillary leak, and reduced serum and lung IL-8 levels after either ischaemic AKI or bilateral nephrectomy ${ }^{113}$. Studies to clarify the mechanism by which IL- 6 causes lung injury in AKI have indicated that circulating, but not pulmonary, IL-6 mediates lung inflammation in $\mathrm{AKI}^{127}$. Intravenous injection of IL- 6 to $\mathrm{Il}^{-6^{-/}}$mice with AKI restores lung inflammation, supporting the hypothesis that circulating IL-6 mediates lung inflammation during $\mathrm{AKI}^{106}$. In contrast, intrapulmonary administration of IL-6 (via intratracheal instillation) does not affect lung inflammation in normal mice or in mice with $\mathrm{AKI}^{127}$. Furthermore, serum IL-6 levels are markedly increased in AKI compared to controls, whereas lung ${ }^{106}$ and BAL fluid levels of IL-6 are low ${ }^{127}$.

IL-6 elicits differential effects depending on the biological context, with the ability to be beneficial or injurious ${ }^{128,129}$. Accumulating data suggest that IL-6 is injurious in models of indirect lung injury (such as haemorrhagic shock ${ }^{130}$ or $\mathrm{AKI}^{105}$ ) yet protective in models of direct lung injury (such as ventilatorinduced lung injury ${ }^{131}$ or with aerosolized and/or inhaled endotoxin ${ }^{127,132}$ ). Thus, circulating IL-6 might be proinflammatory, and intrapulmonary IL- 6 might be anti-inflammatory. Studies indicate that circulating IL-6 mediates lung inflammation in AKI through the upregulation of IL-8 on lung endothelial cells, which promotes neutrophil accumulation ${ }^{106}$. In vitro, addition of IL-6 with soluble IL-6 receptor to endothelial cells increases IL-8 production ${ }^{106}$. Thus, circulating IL-6 likely binds to circulating soluble IL- 6 receptor and signals at endothelial cells via gp130 to increase IL-8 production during AKI (FIG. 3) ${ }^{106}$.

Serum IL-6 and other cytokines accumulate during AKI due to increased renal and extrarenal production, and impaired renal clearance. AKI is characterized by injury to kidney tissue, and renal cytokine production due to injury (either ischaemic or nephrotoxic) has traditionally been presumed as the inciting event that leads to serum cytokine accumulation ${ }^{133}$. mRNA and/or protein of multiple cytokines, including IL-6, have been detected in the kidney after ischaemic or cisplatininduced $\mathrm{AKI}^{89,134-136}$. Studies have demonstrated that cytokines, including IL- 6 , are increased in the serum of mice with bilateral nephrectomy - contradicting the idea that the injured kidney is the sole source of serum cytokine elevation in AKI. In this scenario there is no kidney injury and no kidneys to account for the increase in serum IL-6, suggesting that increased extrarenal production and/or impaired renal clearance contributes to increased serum IL-6 levels after AKI; indeed, data suggest that both conditions are present (FIG. 3) ${ }^{114,133,137}$. Specifically, mRNA levels of $I l-6$ increase in the spleen and liver after bilateral nephrectomy. Macrophage depletion reduces serum IL-6 in mice with bilateral nephrectomy, indicating that macrophages are a key source of cytokine production in $\mathrm{AKI}^{133}$. Studies have also demonstrated that the kidneys are important for cytokine clearance, as intravenous administration of IL-6 is associated with higher serum IL-6 levels in bilaterally nephrectomized mice than in control mice with normal kidney function ${ }^{133}$. Data suggest that IL-6 is normally filtered, and then resorbed and metabolized in the proximal tubule ${ }^{137}$. Interestingly, mice with pre-renal azotaemia and uninjured proximal tubules are able to effectively clear intravenous IL-6, unlike mice with ischaemic AKI and injured proximal tubules ${ }^{137}$.

\section{IL-8 in AKI-mediated lung injury}

IL-8 is a cytokine and neutrophil chemokine that is well studied in human and animal models of ALI, AKI, and AKI-mediated lung injury. IL-8 is increased in the serum and BAL fluid of patients with ALI, and is a predictor of increased mortality ${ }^{125,138}$. Inhibition of IL-8 is protective in numerous animal models of $\mathrm{ALI}^{80,139,140}$. IL-8 is increased in the serum of patients with AKI in whom it is a predictor of increased mortality ${ }^{75}$. Furthermore, increased IL- 8 in patients at $2 \mathrm{~h}$ after the onset of AKI predicts prolonged mechanical ventilation ${ }^{47}$. In mouse models of AKI, both serum and lung IL-8 levels are increased and protection against AKI-mediated lung injury is associated with reduced lung IL-8 levels ${ }^{105,106,108,114}$. The most convincing evidence for a role of IL-8 in AKI-mediated lung injury has come from studies that have demonstrated that mice treated with either IL-8 antibodies or that are deficient in the IL-8 receptor are protected against AKI-mediated lung injury ${ }^{106}$.

\section{TNFR1 and caspase-3 dependent apoptosis}

Programmed cell death via apoptosis contributes to lung injury in ALI, and the role of apoptosis in AKImediated lung injury has been extensively investigated $^{109,112,118,141}$. Gene chip analysis of whole lungs revealed that multiple genes associated with apoptosis 
are increased $6 \mathrm{~h}$ and $36 \mathrm{~h}$ after AKI onset ${ }^{118}$. Multiple lines of evidence have shown that apoptosis occurs in the lung at $24 \mathrm{~h}^{109,112,118,141}$, but not $4 \mathrm{~h}^{109}$ after AKI onset, as judged by increased TUNEL staining and increased caspase- 3 activation in the lung. Caspase- 3 is the predominant executioner caspase that is critical for apoptosis to occur. Colocalization experiments have shown that apoptosis occurs in lung endothelial cells, but not epithelial cells ${ }^{118}$. These in vivo results have been confirmed in vitro by the detection of apoptosis in isolated rat microvascular cells after AKI and in lung microvascular cells incubated with AKI serum ${ }^{112}$. Measures to inhibit apoptosis by pan-caspase inhibition ${ }^{118}$, TNF inhibition (via etanercept) ${ }^{109}$, or TNFR1 deficiency ${ }^{109,118}$ result in less apoptosis and reduced BAL fluid protein, compared to vehicle treatment. In addition to affecting apoptosis, pan-caspase and TNFR1 inhibition might also have beneficial anti-inflammatory effects. Pancaspase inhibition might inhibit activation of proinflammatory caspase-1, and inhibition of TNFR1 might inhibit the action of the proinflammatory cytokine TNF. In summary, the weight of evidence suggests that lung endothelial apoptosis after AKI leads to endothelial cell death, loss of barrier function, and non-cardiogenic pulmonary oedema.

\section{Inflammatory cell mediators}

Of the leucocyte populations that might cause lung injury, only $\mathrm{T}$ lymphocytes have thus far been adequately analyzed and convincingly found to have a causative role in AKI-mediated lung injury. Specifically, $\mathrm{T}$-cell recruitment to the lung increases by $24 \mathrm{~h}$ after AKI, T-cell deficient mice exhibit reduced caspase- 3 activation and less pulmonary oedema compared to control mice, and reconstitution of $\mathrm{T}$ cells can elicit lung injury in T-cell-deficient mice, as judged by increased caspase- 3 activation and increased pulmonary oedema ${ }^{122}$. T cells seem to facilitate lung endothelial apoptosis via caspase-3, leading to lung endothelial cell death and non-cardiogenic pulmonary oedema ${ }^{122}$.

The role of other leucocytes, such as neutrophils and macrophages, in AKI-mediated lung injury is less clear. To date, no studies have examined the effects of neutrophil depletion on lung injury after AKI, and studies have only assessed neutrophil infiltration as a marker of lung injury after AKI. Two reports have examined the role of macrophages in AKI-mediated lung injury ${ }^{123}$. In one report, macrophage function was inhibited using CNI-1493 in a rat model of ischaemic AKI and capillary leak was subsequently reduced (inflammation was not measured $)^{35}$. In another report, depletion of either alveolar macrophages or systemic macrophages demonstrated mixed results in showing that lung inflammation was reduced yet capillary leak was increased. Many explanations might underlie these apparently discrepant results, including an injurious effect of the macrophage depletion method itself on capillary leak. The role of macrophages in AKI-mediated lung injury, therefore, remains unresolved, although the data do suggest that both systemic and alveolar macrophages mediate lung inflammation after AKI.

\section{Lung injury beyond $\mathbf{4 8 h}$}

Only one study has examined the effects of AKI on the lung beyond $48 \mathrm{~h}^{142}$. In this study, four fluid strategies were tested in mice to assess the effect of fluid administration on kidney function and lung inflammation at $24 \mathrm{~h}$ and 7 days after AKI. The fluid strategies comprised $100 \mu \mathrm{l}, 500 \mu \mathrm{l}, 1,000 \mu \mathrm{l}$, or $2,000 \mu \mathrm{l}$ of $0.9 \%$ normal saline, administered subcutaneously every day from the day of surgery to induce ischaemic AKI. Adult mice generally weigh $20-25 \mathrm{~g}$, thus $2,000 \mu \mathrm{l}$ fluid $(2 \mathrm{~g})$ is $\sim 10 \%$ of the body weight and approximately two-times the blood volume of an adult mouse. The intent of this experiment was to assess the effect of fluid overload in lung injury after AKI. The researchers found no difference in AKI or lung injury among the different fluid strategies at $24 \mathrm{~h}$ after AKI, but the mice that received 2,000 $\mu$ l fluid daily showed less AKI and lung inflammation 7 days after surgery, compared to those that received $100 \mu$ fluid daily. Thus, the highest volume of fluid administration hastened both the recovery of kidney function and the resolution of lung inflammation. Fluid overload did not occur because mice with recovering kidney function simply excreted the excess fluid in urine.

These data suggest a number of key, clinically relevant points: early and aggressive fluid administration can reduce the duration of ischaemia-induced AKI; delayed resolution of AKI results in lung neutrophil accumulation and inflammation at 7 days post AKI; reducing the duration of AKI reduces lung inflammation; and lung inflammation post-AKI is not dependent on fluid overload. These data are consistent with the accumulating evidence suggesting that the adverse outcomes and increased mortality of AKI are associated with duration of $\mathrm{AKI}^{143,144}$. These data are also in line with current clinical practice that emphasizes early volume resuscitation in the setting of hypoperfusion, such as sepsis ${ }^{145}$. Appropriate fluid administration led to a notable improvement in the rate of kidney function recovery even in a mouse model of ischaemia-induced AKI that was generated by clamping the renal artery and vein ${ }^{142}$. On the other hand, fluid overload in AKI has well-known associations with deleterious consequences. Thus, when facing a patient with AKI, the clinician must carefully assess volume status and haemodynamics. Early AKI with hypovolaemia merits appropriate volume challenge. In late AKI, when injury is likely established, a volume challenge might be less effective and the clinician should be mindful to avoid fluid overload.

\section{Conclusions}

AKI is common in hospitalized patients and is associated with increased mortality. To date, animal research in AKI has predominantly focused on elucidating the pathophysiology of kidney injury. Therapeutic studies in animal models have predominantly tested preventative interventions given prior to the onset of AKI, or at the time of AKI induction ${ }^{146}$. Clinical trials in AKI have also focused on measures to prevent $\mathrm{AKI}^{146}$, although the effect of modifications of RRT has also been widely studied ${ }^{146}$. To date, however, no specific therapy has been shown to effectively prevent AKI in patients, and the 
mortality of patients with AKI requiring RRT remains $>50 \%$ in the ICU. We suggest that reducing the mortality of patients with AKI requires a shift in pre-clinical and clinical research from prevention to the later stages of AKI (beyond $24 \mathrm{~h}$ ) when the disease is most likely to be recognized.

As discussed in this Review, mounting data indicate that $\mathrm{AKI}$ is a systemic disease that has deleterious effects on the immune system and numerous organs, including the lung. Data so far suggest that these complications contribute to the high mortality of patients with AKI and are unlikely to be corrected solely by dialysis. Thus, we believe that additional studies of the mechanisms of systemic organ injury have great potential to reduce the overall mortality of patients with AKI and that additional basic and clinical research into this area is needed.
Respiratory complications are particularly common in patients with AKI and greatly increase mortality. Respiratory complications are predominantly due to pulmonary oedema, which might be cardiogenic or non-cardiogenic. Animal data indicate that lung inflammation and lung endothelial apoptosis mediate noncardiogenic pulmonary oedema after AKI. Although numerous mediators of lung injury after AKI have been identified (BOX 3), these mediators all affect lung injury within $24 \mathrm{~h}$. Thus, future studies must focus on the later pulmonary consequences of AKI, as well as other systemic effects, in order to better facilitate the translation of basic research to patients with AKI. Measures to reduce respiratory, and other systemic, non-traditional complications of AKI hold great promise to reduce the very high mortality of patients with AKI.
1. Uchino, S. et al. An assessment of the RIFLE criteria for acute renal failure in hospitalized patients. Crit. Care Med. 34, 1913-1917 (2006).

2. Star, R. A. Treatment of acute renal failure. Kidney Int 54, 1817-1831 (1998)

3. Koyner, J. L. et al. The daily burden of acute kidney injury: a survey of, US nephrologists on World Kidney Day. Am. J. Kidney Dis. 64, 394-401 (2014).

4. Xue, J. L. et al. Incidence and mortality of acute renal failure in Medicare beneficiaries, 1992 to 2001. J. Am. Soc. Nephrol. 17, 1135-1142 (2006).

5 Uchino, S. et al. Continuous renal replacement therapy: a worldwide practice survey. The beginning and ending supportive therapy for the kidney (B.E.S.T kidney) investigators. Intensive Care Med. 33 , 1563-1570 (2007).

6. VA/NIH Acute Renal Failure Trial Network, Palevsky, P. M., Zhang, J. H. et al. Intensity of renal support in critically ill patients with acute kidney injury. N. Engl. J. Med. 359, 7-20 (2008).

7. Chertow, G. M. et al. Acute kidney injury, mortality, length of stay, and costs in hospitalized patients. J. Am. Soc. Nephrol. 16, 3365-3370 (2005).

8. Liangos, O. et al. Epidemiology and outcomes of acute renal failure in hospitalized patients: a national survey. Clin. J. Am. Soc. Nephrol. 1, 43-51 (2006).

9. Levy, E. M., Viscoli, C. M. \& Horwitz, R. I. The effect of acute renal failure on mortality. A cohort analysis. JAMA 275, 1489-1494 (1996).

10. Bates, D. W. et al. Mortality and costs of acute renal failure associated with amphotericin B therapy. Clin. Infect. Dis. 32, 686-693 (2001)

11. Chertow, G. M. et al. Independent association between acute renal failure and mortality following cardiac surgery. Am. J. Med. 104, 343-348 (1998).

12. Lassnigg, A. et al. Minimal changes of serum creatinine predict prognosis in patients after cardiothoracic surgery: a prospective cohort study. J. Am. Soc. Nephrol. 15, 1597-1605 (2004).

13. Parikh, C. R. et al. Renal dysfunction in allogeneic hematopoietic cell transplantation. Kidney Int 62 566-573 (2002)

14. Metnitz, P. G. et al. Effect of acute renal failure requiring renal replacement therapy on outcome in critically ill patients. Crit. Care Med. 30, 2051-2058 (2002).

15. du Cheyron, D. et al. The attributable mortality of acute renal failure in critically ill patients with liver cirrhosis. Intensive Care Med. 31, 1693-1699 (2005).

16. Hoste, E. \& DeCorte, W. D. Clinical Consequences of Acute Kidney Injury. Contrib. Nephrol. 174, 56-64 (2011).

17. Okusa, M. \& Rosner, M. H. Overview of the management of acute kidney injury (acute renal failure). In: UpToDate. Palevsky, P. M. (ed.), UpToDate, Waltham, MA. (2014).

18. Macedo, E., Bouchard, J. \& Mehta, R. in Comprehensive Clinical Nephrology. $5^{\text {th }}$ edn Ch. 14 (eds Johnson, R. J., Feehally, J. \& Floege, J.) 851-853 (Saunders, 2014).

19. KDIGO. KDIGO clinical practice guideline for acute kidney injury. Kidney Int. Suppl. 2, 1-138 (2012).
20. Murugan, R. \& Kellum, J. A. Acute kidney injury: what's the prognosis? Nat. Rev. Nephrol. 7, 209-217 (2011).

21. Macedo, E. \& Mehta, R. L. in Schrier's Diseases of the Kidney. $9^{\text {th }}$ edn Ch. 28. (eds Schrier, R. W. Coffman, T. M., Falk, R. J., Molitoris, B. A. \& Neilson, E. G.) 795-825 (LWW, 2012)

22. Palevsky, P. Renal replacement therapy (dialysis) in acute kidney injury (acute renal failure) in adults: indications, timing, and dialysis dose. In: UpToDate. Burns, J. \& Sheridan, A. M. (eds), UpToDate, Waltham, MA. (2013).

23. Teschan, P. E. et al. Prophylactic hemodialysis in the treatment of acute renal failure. Ann. Intern. Med. 53, 992-1016 (1960)

24. Frank, H. A., Seligman, A. M. \& Fine, J. Further experiences with peritoneal irrigation for acute renal failure: including a description of modifications in method. Ann. Surg. 128, 561-608 (1948).

25. Wacker, W. \& Merrill, J. P. Uremic pericarditis in acute and chronic renal failure. JAMA 156, 764-765 (1954).

26. Cahalane, S. F. et al. Acquired thrombocytopathy: observations on the coagulation defect in uremia. Am. J. Clin. Pathol. 30, 507-513 (1958)

27. Bagshaw, S. M. et al. Fluid balance as a biomarker: impact of fluid overload on outcome in critically ill patients with acute kidney injury. Crit. Care. 12, 169 (2008).

28. Bouchard, J. et al. Fluid accumulation, survival and recovery of kidney function in critically ill patients with acute kidney injury. Kidney Int. 76, 422-427 (2009).

29. Vaara, S. T. et al. Fluid overload is associated with an increased risk for 90-day mortality in critically ill patients with renal replacement therapy: data from the prospective FINNAKI study. Crit. Care 16, R197 (2012).

30. Mehta, R. L. et al. Sepsis as a cause and consequence of acute kidney injury: Program to Improve Care in Acute Renal Disease. Intensive Care Med. 37 241-248 (2011).

31. Ronco, C., House, A. A. \& Haapio, M. Cardiorenal syndrome: refining the definition of a complex symbiosis gone wrong. Intensive Care Med. 34 957-962 (2008)

32. Waikar, S. S., Liu, K. D. \& Chertow, G. M. The incidence and prognostic significance of acute kidney injury. Curr. Opin. Nephrol. Hypertens. 16, 227-236 (2007).

33. Himmelfarb, J. et al. Impaired monocyte cytokine production in critically ill patients with acute renal failure. Kidney Int. 66, 2354-2360 (2004).

34. Hassoun, H. T. et al. Ischemic acute kidney injury induces a distant organ functional and genomic response distinguishable from bilateral nephrectomy. Am. J. Physiol Renal Physiol 293, F30-F40 (2007).

35. Kramer, A. A. et al. Renal ischemia/reperfusion leads to macrophage-mediated increase in pulmonary vascular permeability. Kidney Int. 55, 2362-2367 (1999)

36. Kelly, K. J. Distant effects of experimental renal ischemia/reperfusion injury. J. Am. Soc. Nephrol. 14 1549-1558 (2003).
37. Kim, M. et al. Isoflurane activates intestinal sphingosine kinase to protect against bilateral nephrectomy-induced liver and intestine dysfunction. Am. J. Physiol. Renal Physiol. 300, F167-F176 (2011).

38. Yildirim, A. et al. Dehydroepiandrosterone improves hepatic antioxidant systems after renal ischemiareperfusion injury in rabbits. Ann. Clin. Lab. Sci. 33 459-464 (2003)

39. Liu, M. et al. Acute kidney injury leads to inflammation and functional changes in the brain. J. Am. Soc. Nephrol. 19, 1360-1370 (2008).

40. Park, S. W. et al. Paneth cell-mediated multiorgan dysfunction after acute kidney injury. J. Immunol. 189 5421-5433 (2012)

41. Scheel, P. J., Liu, M. \& Rabb, H. Uremic lung: new insights into a forgotten condition. Kidney Int. 74 849-851 (2008)

42. Kelly, K. J. Acute renal failure: much more than a kidney disease. Semin. Nephrol. 26, 105-113 (2006)

43. Awad, A. S. \& Okusa, M. D. Distant organ injury following acute kidney injury. Am. J. Physiol. Renal Physiol. 293, F28-29 (2007).

44. Faubel, S. Acute kidney injury and multiple organ dysfunction syndrome. Minerva Urol. Nefrol. 61, 171-188 (2009).

45. Faubel, S. Pulmonary complications after acute kidney injury. Adv. Chronic Kidney Dis. 15, 284-296 (2008).

46. Imai, Y. et al. Injurious mechanical ventilation and end organ epithelial cell apoptosis and organ dysfunction in an experimental model of acute respiratory distress syndrome. JAMA 289, 2104-2112 (2003).

47. Liu, K. D. et al. Serum interleukin-6 and interleukin-8 are early biomarkers of acute kidney injury and predict prolonged mechanical ventilation in children undergoing cardiac surgery: a case-control study. Crit. Care 13, R104 (2009).

48. Bhargava, R. et al. Acute lung injury and acute kidney injury are established by four hours in experimental sepsis and are improved with pre, but not post, sepsis administration of TNF- $\alpha$ antibodies. PLOS ONE 8, e79037 (2013)

49. Chertow, G. M. et al. Prognostic stratification in critically ill patients with acute renal failure requiring dialysis. Arch. Intern. Med. 155,1505-1511 (1995).

50. Mehta, R. L. et al. Refining predictive models in critically ill patients with acute renal failure. J. Am Soc. Nephrol. 13, 1350-1357 (2002).

51. Neveu, H. et al. Prognostic factors in acute renal failure due to sepsis. Results of a prospective multicentre study. The French Study Group on Acute Renal Failure. Nephrol. Dial. Transplant. 11, 293-299 (1996).

52. Uchino, S. et al. Acute renal failure in critically ill patients: a multinational, multicenter study. JAMA 294, 813-818 (2005).

53. Lins, R. L. et al. Re-evaluation and modification of the Stuivenberg Hospital Acute Renal Failure (SHARF) scoring system for the prognosis of acute renal failure: an independent multicentre, prospective study. Nephrol. Dial. Transplant. 19, 2282-2288 (2004). 
54. Chertow, G. M. et al. Predictors of mortality and the provision of dialysis in patients with acute tubular necrosis. The Auriculin Anaritide Acute Renal Failure Study Group. J. Am. Soc. Nephrol. 9, 692-698 (1998).

55. Paganini, E. P., Halstenberg, W. K. \& Goormastic, M. Risk modeling in acute renal failure requiring dialysis: the introduction of a new model. Clin. Nephrol. 46, 206-211 (1996)

56. Liano, F. et al. Prognosis of acute tubular necrosis: an extended prospectively contrasted study. Nephron 63 21-31 (1993)

57. Demirjian, S. et al. Model to predict mortality in critically ill adults with acute kidney injury. Clin. J. Am. Soc. Nephrol. 6, 2114-2120 (2011).

58. Pike, F. et al. Biomarker enhanced risk prediction for adverse outcomes in critically ill patients receiving RRT Clin. J. Am. Soc. Nephrol. 10, 1332-1339 (2015).

59. [No authors listed] Ventilation with lower tidal volumes as compared with traditional tidal volumes for acute lung injury and the acute respiratory distress syndrome. The Acute Respiratory Distress Syndrome Network. N. Engl. J. Med. 342, 1301-1308 (2000).

60. Vieira, J. M. et al. Effect of acute kidney injury on weaning from mechanical ventilation in critically ill patients. Crit. Care Med. 35, 184-191 (2007).

61. Grassi, V. et al. Uremic lung. Contrib. Nephrol. 106 36-42 (1994)

62. Alwall, N., Lunderquist, A., \& Olsson, O. Studies on electrolyte-fluid retention. I. Uremic lung, fluid lung? On pathogenesis and therapy; a preliminary report. Acta Med. Scand. 146, 157-163 (1953).

63. Kwiatkowski, D. M. et al. Improved outcomes with peritoneal dialysis catheter placement after cardiopulmonary bypass in infants. J. Thorac Cardiovasc. Surg. 149, 230-236 (2015).

64. Payen, D. et al. A positive fluid balance is associated with a worse outcome in patients with acute renal failure. Crit. Care 12, R74 (2008).

65. Bouchard, J. et al. Fluid accumulation, survival and recovery of kidney function in critically ill patients with acute kidney injury. Kidney Int. 76, 422-427 (2009).

66. Matthay, M. A., Ware, L. B. \& Zimmerman, G. A. The acute respiratory distress syndrome. J. Clin. Invest. 122, 2731-2740 (2012)

67. Rackow, E. C., Fein, I. A., Sprung, C. \& Grodman, R. S. Uremic pulmonary edema. Am. J. Med. 64, 1084-1088 (1978).

68. Gibson, D. G. Haemodynamic factors in the development of acute pulmonary oedema in renal failure. Lancet 2, 1217-1220 (1966).

69. Matthay, M. A. \& Zimmerman, G. A. Acute lung injury and the acute respiratory distress syndrome: four decades of inquiry into pathogenesis and rational management. Am. J. Respir. Cell. Mol. Biol. 33, 319-327 (2005)

70. Zettergren, L. Uremic lung; report of four cases reaching autopsy. Acta Soc. Med. Ups. 60, 161-171 (1955).

71. Hopps, H. C. \& Wissler, R. W. Uremic pneumonitis. Am. J. Pathol. 31, 261-273 (1955)

72. Roubier, C. \& Plauchau, M. Sur certains aspects radiographiques de l'oœdème pulmonaire chez les cardiorénaux azotémiques [French]. Arch. Méd-chir de l'app. Respire. 9, 189 (1934).

73. Bass, H. E. et al. Pulmonary changes in uremia. JAMA 148, 724-726 (1952)

74. Bleyl, U., Sander, E. \& Schindler, T. The pathology and biology of uremic pneumonitis. Intensive Care Med. 7 193-202 (1981)

75. Simmons, E. M. et al. Plasma cytokine levels predict mortality in patients with acute renal failure. Kidney Int. 65, 1357-1365 (2004).

76. Chawla, L. S. et al. Elevated plasma concentrations of IL- 6 and elevated APACHE II score predict acute kidney injury in patients with severe sepsis. Clin. J. Am Soc. Nephrol. 2, 22-30 (2007).

77. Zhang, W. R. et al. Plasma IL- 6 and IL-10 concentrations predict AKI and long-term mortality in adults after cardiac surgery. J. Am. Soc. Nephrol. http://dx.doi.org/ASN.2014080764.

78. Murugan, R. et al. Plasma inflammatory and apoptosis markers are associated with dialysis dependence and death among critically ill patients receiving renal replacement therapy. Nephrol. Dial. Transplant. 29, 1854-1864 (2014).

79. Liangos, O. et al. Interleukin-8 and acute kidney injury following cardiopulmonary bypass: a prospective cohort study. Nephron Clin. Pract. 113, c148-c154 (2009).
80. Bless, N. M. et al. Roles for C-X-C chemokines and C5a in lung injury after hindlimb ischemia-reperfusion. Am. J. Physiol. 276, L57-63 (1999).

81. Colletti, L. M. et al. Tumor necrosis factor up-regulates intercellular adhesion molecule 1, which is important in the neutrophil-dependent lung and liver injury associated with hepatic ischemia and reperfusion in the rat. Shock 10, 182-191 (1998).

82. Colletti, L. M. et al. Chemokine expression during hepatic ischemia/reperfusion-induced lung injury in the rat The role of epithelial neutrophil activating protein. J. Clin. Invest. 95, 134-141 (1995)

83. Seekamp, A. et al. Role of beta 2 integrins and ICAM-1 in lung injury following ischemia-reperfusion of rat hind limbs. Am. J. Pathol. 143, 464-472 (1993).

84. Yoshidome, H., Kato, A., Edwards, M. J. \& Lentsch, A. B. Interleukin-10 inhibits pulmonary NF-kappaB activation and lung injury induced by hepatic ischemiareperfusion. Am. J. Physiol. 277, L919-923 (1999).

85. Yoshidome, $\mathrm{H}$. et al. Enhanced pulmonary expression of CXC chemokines during hepatic ischemia/ reperfusion-induced lung injury in mice. J. Surg. Res. 81, 33-37 (1999)

86. Depass, S. W. et al. Pulmonary congestion and edema in uremia. JAMA 162, 5-9 (1956).

87. Kuebler, W. M. et al. Pressure is proinflammatory in lung venular capillaries. J. Clin. Invest. 104, 495-502 (1999).

88. Wiedemann, H. P. et al. Comparison of two fluidmanagement strategies in acute lung injury. N. Engl. J. Med. 354, 2564-2575 (2006).

89. Hoke, T. S. et al. Acute renal failure after bilateral nephrectomy is associated with cytokine-mediated pulmonary injury. J. Am. Soc. Nephrol. 18, 155-164 (2007).

90. Clermont, G. et al. Renal failure in the ICU: comparison of the impact of acute renal failure and end-stage renal disease on ICU outcomes. Kidney Int 62, 986-996 (2002).

91. Knaus, W. A. et al. The APACHE III prognostic system. Risk prediction of hospital mortality for critically ill hospitalized adults. Chest 100, 1619-1636 (1991).

92. Ostermann, M. \& Chang, R. Renal failure in the intensive care unit: acute kidney injury compared to end-stage renal failure. Crit. Care 12, 432 (2008).

93. Rocha, E. et al. Outcomes of critically ill patients with acute kidney injury and end-stage renal disease requiring renal replacement therapy: a case-control study. Nephrol. Dial. Transplant. 24, 1925-1930 (2009).

94. Hussain, S. et al. Outcome among patients with acute renal failure needing continuous renal replacement therapy: a single center study. Hemodial. Int. 13, 205-214 (2009).

95. Walcher, A. et al. In critically ill patients requiring CRRT, AKI is associated with increased respiratory failure and death versus ESRD. Ren. Fail. 33 , 935-942 (2011).

96. Kramer, H. J. et al. Increasing body mass index and obesity in the incident ESRD population. J. Am. Soc. Nephrol. 17, 1453-1459 (2006)

97. Bellingan, G. J. The pulmonary physician in critical care *6: The pathogenesis of ALI/ARDS. Thorax 57 540-546 (2002).

98 Chonchol, M. Neutrophil dysfunction and infection risk in end-stage renal disease. Semin. Dial. 19, 291-296 (2006)

99. Tsalik, E. L. et al. Renal systems biology of patients with systemic inflammatory response syndrome. Kidney Int. http://dx.doi.org/10.1038/ki.2015.150.

100. Matute-Bello, G. et al. An official American Thoracic Society workshop report: features and measurements of experimental acute lung injury in animals. $A m$. J. Respir. Cell Mol. Biol. 44, 725-738 (2011).

101. Tremblay, L. et al. Injurious ventilatory strategies increase cytokines and c-fos m-RNA expression in an isolated rat lung model. J. Clin. Invest. 99, 944-952 (1997).

102. Hu, G. et al. Intercellular adhesion molecule-1-dependent neutrophil adhesion to endothelial cells induces caveolae-mediated pulmonary vascular hyperpermeability. Circ. Res. 102 e120-131 (2008)

103. Zarbock, A. et al. Acute uremia but not renal inflammation attenuates aseptic acute lung injury: a critical role for uremic neutrophils. J. Am. Soc Nephrol. 17, 3124-3131 (2006).

104. Deng, J., Hu, X., Yuen, P. S. \& Star, R. A. Alphamelanocyte-stimulating hormone inhibits lung injury after renal ischemia/reperfusion. Am. J. Respir. Crit. Care Med. 169, 749-756 (2004).
105. Klein, C. L. et al. Interleukin-6 mediates lung injury following ischemic acute kidney injury or bilateral nephrectomy. Kidney Int. 74, 901-909 (2008).

106. Ahuja, N. et al. Circulating IL-6 mediates lung injury via CXCL1 production after acute kidney injury in mice. Am. J. Physiol. Renal Physiol. 303, F864-F872 (2012).

107. Gu, J. et al. Dexmedetomidine attenuates remote lung injury induced by renal ischemia-reperfusion in mice. Acta. Anaesthesiol. Scand. 55, 1272-1278 (2011).

108. Doi, K. et al. The high-mobility group protein B1-Toll-like receptor 4 pathway contributes to the acute lung injury induced by bilateral nephrectomy. Kidney Int. 86, 316-326 (2014).

109. White, L. E. et al. TNFR1-dependent pulmonary apoptosis during ischemic acute kidney injury. Am. J. Physiol. Lung Cell. Mol. Physiol. 303, L449-L459 (2012).

110. Awad, A. S. et al. Compartmentalization of neutrophils in the kidney and lung following acute ischemic kidney injury. Kidney Int. 75, 689-698 (2009).

111. Lee, W. L. \& Downey, G. P. Neutrophil activation and acute lung injury. Curr. Opin. Crit. Care. 7, 1-7 (2001)

112. Feltes, C. M. et al. Pulmonary endothelial cell activation during experimental acute kidney injury. Shock 36, 170-176 (2011).

113. Klein, C. L. et al. Interleukin-6 mediates lung injury following ischemic acute kidney injury or bilateral nephrectomy. Kidney Int. (2008).

114. Andres-Hernando, A. et al. Splenectomy exacerbates lung injury after ischemic acute kidney injury in mice. Am. J. Physiol. Renal Physiol. 301, F907-F916 (2011)

15. Grigoryev, D. N. et al. The local and systemic inflammatory transcriptome after acute kidney injury. J. Am. Soc. Nephrol. 19, 547-558 (2008)

116. Kim do, J. et al. Comparison of experimental lung injury from acute renal failure with injury due to sepsis Respiration 73, 815-824 (2006)

117. Ma, T. \& Liu, Z. Functions of aquaporin 1 and alphaepithelial $\mathrm{Na}+$ channel in rat acute lung injury induced by acute ischemic kidney injury. Int. Urol. Nephrol. 45, 1187-1196 (2013).

118. Hassoun, H. T. et al. Kidney ischemia-reperfusion injury induces caspase-dependent pulmonary apoptosis. Am. J. Physiol. Renal Physiol. 297, F125-F137 (2009)

119. Heidland, A. et al. Uremic pneumonitis. Evidence for participation of proteolytic enzymes. Contrib. Nephrol. 41, 352-366 (1984).

120. Peterson, B. T., Brooks, J. A. \& Hyde, R. W. Lung fluid balance during acute renal failure in sheep. J. Appl. Physiol. 60, 1333-1340 (1986).

121. Rabb, H. et al. Acute renal failure leads to dysregulation of lung salt and water channels. Kidney Int. 63, 600-606 (2003).

122. Lie, M. L. et al. Lung T lymphocyte trafficking and activation during ischemic acute kidney injury. J. Immunol. 189, 2843-2851 (2012).

123. Altmann, C. et al. Macrophages mediate lung inflammation in a mouse model of ischemic acute kidney injury. Am. J. Physiol. Renal Physiol. 302 , F421-F432 (2012)

124. Liu, K. D. et al. Predictive and pathogenetic value of plasma biomarkers for acute kidney injury in patients with acute lung injury. Crit. Care Med. 35 2755-2761 (2007).

125. Meduri, G. U. et al. Inflammatory cytokines in the BAL of patients with ARDS. Persistent elevation over time predicts poor outcome. Chest 108, 1303-1314 (1995).

126. Parsons, P. E. et al. Lower tidal volume ventilation and plasma cytokine markers of inflammation in patients with acute lung injury. Crit. Care Med. 33, 1-6 (2005)

127. Bhargava, R. et al. Intratracheal IL-6 protects against lung inflammation in direct, but not indirect, causes of acute lung injury in mice. PLOS ONE 8, e61405 (2013).

128. Kamimura, D., Ishihara, K. \& Hirano, T. IL-6 signal transduction and its physiological roles: the signal orchestration model. Rev. Physiol. Biochem. Pharmacol. 149, 1-38 (2003).

129. Calabrese, L. H. \& Rose-John, S. IL-6 biology: implications for clinical targeting in rheumatic disease. Nat. Rev. Rheumatol. 10, 720-727 (2014).

130. Meng, Z. H. et al. Essential role for IL-6 in postresuscitation inflammation in hemorrhagic shock. Am. J. Physiol. Cell Physiol. 280, C343-C351 (2001)

131. Wolters, P. J. et al. Neutrophil-derived IL-6 limits alveolar barrier disruption in experimental ventilator induced lung injury. J. Immunol. 182, 8056-8062 (2009). 
132. Xing, Z. et al. IL-6 is an antiinflammatory cytokine required for controlling local or systemic acute inflammatory responses. J. Clin. Invest. 101, 311-320 (1998).

133. Andres-Hernando, A et al. Cytokine production increases and cytokine clearance decreases in mice with bilateral nephrectomy. Nephrol. Dial. Transplant. 27, 4339-4347 (2012).

134. Kielar, M. L. et al. Maladaptive role of IL-6 in ischemic acute renal failure. J. Am. Soc. Nephrol. 16. 3315-3325 (2005).

135. Zhang, Y. et al. Ischemia-reperfusion induces G.-CSF gene expression by renal medullary thick ascending limb cells in vivo and in vitro Am. J. Physiol. Renal Physiol. 286, F1193-F1201 (2004).

136. Faubel, S. et al. Cisplatin-induced acute renal failure is associated with an increase in the cytokines interleukin (IL)-1 beta, IL-18, IL-6, and neutrophil infiltration in the kidney. J. Pharmacol. Exp. Ther 322, 8-15 (2007).
137. Dennen, P. et al. Urine interleukin-6 is an early biomarker of acute kidney injury in children undergoing cardiac surgery. Crit. Care 14, R181 (2010).

138. Meduri, G. U. et al. Persistent elevation of inflammatory cytokines predicts a poor outcome in ARDS. Plasma IL-1 beta and IL- 6 levels are consistent and efficient predictors of outcome over time. Chest 107, 1062-1073 (1995)

139. Belperio, J. A. et al. Critical role for CXCR2 and CXCR2 ligands during the pathogenesis of ventilator-induced lung injury. J. Clin. Invest. 110, 1703-1716 (2002).

140. Puneet, P., Moochhala, S. \& Bhatia, M. Chemokines in acute respiratory distress syndrome. Am. J. Physiol. Lung Cell. Mol. Physiol. 288, L3-15 (2005).

141. White, L. E. et al. Lung endothelial cell apoptosis during ischemic acute kidney injury. Shock 38 320-327 (2012).

142. Andres-Hernando, A. et al. Prolonged acute kidney injury exacerbates lung inflammation at 7 days postacute kidney injury. Physiol. Rep. 2, e12084 (2014).
143. Palevsky, P. M. et al. KDOQI US commentary on the KDIGO clinical practice guideline for acute kidney injury. Am. J. Kidney Dis. 61, 649-672 (2013).

144. Coca, S. G. et al. The duration of postoperative acute kidney injury is an additional parameter predicting long-term survival in diabetic veterans. Kidney Int. 78 926-933 (2010)

145. Rivers, E. et al. Early goal-directed therapy in the treatment of severe sepsis and septic shock. N. Engl. J. Med. 345, 1368-1377 (2001).

146. Faubel, S. et al. Ongoing clinical trials in AKI. Clin. J. Am. Soc. Nephrol. 7, 861-873 (2012).

\section{Author contributions}

S.F. researched the data for the article. S.F. and C.L.E. provided substantial contributions to discussions of its content, wrote the article and undertook review and/or editing of the manuscript before submission.

Competing interests statement

The authors declare no competing interests. 